\title{
Biology of consciousness
}

\section{Gerald M. Edelman*, Joseph A. Gally and Bernard J. Baars}

The Neurosciences Institute, San Diego, CA, USA

Edited by:

Morten Overgaard, Aarhus University,

Denmark

Reviewed by:

Alexander Maier, National Institute for Mental Health, USA

*Correspondence:

Gerald M. Edelman, The

Neurosciences Institute, 10640 John

Jay Hopkins Drive, San Diego, CA

92121, USA.

e-mail:edelman@nsi.edu

\begin{abstract}
The Dynamic Core and Global Workspace hypotheses were independently put forward to provide mechanistic and biologically plausible accounts of how brains generate conscious mental content. The Dynamic Core proposes that reentrant neural activity in the thalamocortical system gives rise to conscious experience. GlobalWorkspace reconciles the limited capacity of momentary conscious content with the vast repertoire of long-term memory. In this paper we show the close relationship between the two hypotheses. This relationship allows for a strictly biological account of phenomenal experience and subjectivity that is consistent with mounting experimental evidence. We examine the constraints on causal analyses of consciousness and suggest that there is now sufficient evidence to consider the design and construction of a conscious artifact.
\end{abstract}

Keywords: Global Workspace, Dynamic Core, Neural Darwinism, reentry, qualia

\section{INTRODUCTION}

Advances in neuroscience have now made it possible to study the biological basis of consciousness. Indeed, in recent years an increasing amount of attention has been directed to this subject (Crick and Koch, 2003; Edelman, 2003; Velmans and Schneider, 2007; Zelazo et al., 2007). Our own efforts to account for key aspects of consciousness at a biological level have taken two forms. The first involved the proposal of a neuroscientifically based global brain theory commonly referred to as Neural Darwinism (Edelman, 1978, 1987; Edelman and Tononi, 2000). This theory proposes the functioning of a Dynamic Core generated by a neural process, reentry, to link dispersed cortical and thalamic areas and account for the relation between perception and conscious memory. The second theory (Baars, 1988) was propounded mainly from a cognitive psychological point of view. This Global Workspace theory hypothesizes that a number of brain components constitute an integrative workspace that serves to reconcile the narrow momentary capacity of conscious contents with a widespread recruitment of unconscious brain functions, including long-term memory.

In the present account, we reconcile and expand on these early notions by considering consciousness as a biological phenomenon, one that is a product of both evolution and development. We believe that such a biological approach can address and even dispose of several concerns articulated by philosophers of mind and others. We propose that a biological account of consciousness does not require metaphysical proposals, mathematical reduction, or "strange physics." We also maintain that previously argued categories such as selfhood and phenomenal experience can be explained biologically in terms of patterns of neural activity.

At the outset, it is important to distinguish primary sensorimotor consciousness from higher-order consciousness (Edelman, 1992, 2003). Primary consciousness occurs in animals lacking any linguistic capabilities, and is an essential process even in humans. After the invention of language it was possible to escape the "remembered present" of primary consciousness by referring to internal linguistic tokens. As a result, possession of true language with syntax in humans gives rise to higher-order consciousness allowing its possessor to be conscious of being conscious. We emphasize this distinction to make the point that our overarching task here is to account in biological terms for primary consciousness. At the same time, such an account necessarily depends on investigation of human subjects, with their ability verbally to report, as the richest source of relevant data.

\section{A SUMMARY REVIEW}

In the last decade a large number of observations and experiments have contributed to our understanding of consciousness (see for example, Velmans and Schneider, 2007; Zelazo et al., 2007). Here, our aim is to consider certain salient aspects of this work.

In the vertebrates, consciousness is a dynamic, integrated, multimodal mental process entailed by physical events occurring in the forebrain. Plausibly, the neural structures and mechanisms underlying consciousness were selected during evolution because they allowed animals to plan and prepare for future eventualities. Conscious processes arise spontaneously and display intentionality, i.e., for the most part, each is about something (Brentano, 1973). At any given moment, this content comprises a unified "scene" that can be shaped either by a limited selection of exogenous sensory input, by endogenous constructs generated from stored memories, or by some combination of the two. Consciousness is necessarily subjective and internal (Metzinger, 2003), but its contents can often be inferred from animal behavior or verbal report. In humans the contents of consciousness are substantially under autonomous regulation, i.e., we decide where to direct our sensory attention or thought processes (Knudsen, 2007). Consciousness of acts or feelings seems to be essential for the laying down of long-term episodic memories (Tulving, 1987).

Much evidence shows that, in mammals, the neural structures required for specific conscious events include the thalamus and cerebral cortex. For example, researchers using brain-imaging techniques, e.g., fMRI (Norman et al., 2006) or magnetoencephalography (MEG; Srinivasan et al., 1999), can often deduce with a significant degree of success the general nature of the contents of a subject's consciousness from the anatomic and temporal 
distribution of neural activity generated in the subjects' cortex. This is the case regardless of whether these contents are evoked endogenously or by sensory input (Kanwisher, 2010). Moreover, lesions in the thalamus or cortex, or disruptions of their normal functioning through various techniques, can disrupt the contents of consciousness (Bogen, 1995, 1997).

Nevertheless, not all patterns of neural activity in the thalamus or cortex relate to, or are necessary for consciousness. Inactivation or removal of large portions of these structures does not necessarily render an animal unconscious. Consciousness can also persist following the loss of large brain components, such as the hippocampus (Milner et al., 1968), the cerebellum (Verschuuren et al., 1996), the frontal cortex (Stookey et al., 1941), or even an entire cerebral hemisphere (Austin and Grant, 1956). By contrast, the thalamic intralaminar nuclei, which project axons widely to all cortical areas, are notable in that their destruction can result in a permanent loss of consciousness (Bogen, 1997).

The corticothalamic system of any living animal displays spontaneous patterns of neural activity whether that animal is conscious or not. From temporal correlations among fluctuations in such activity in various regions of the brain, one can infer the presence of networks mediating functional connectivity among these regions (Fox and Raichle, 2007). Anatomic networks of long-range axonal bundles allow this connectivity by providing critical bi-directional reentrant links among cortical areas and thalamic nuclei (Honey et al., 2009).

Microelectrode, EEG, and MEG measurements all support the hypothesis that when separate cortical areas contribute to the contents of consciousness, they exhibit enhanced synchrony in the gamma frequency band (Engel and Singer, 2001) that may be phaselocked to a slower theta rhythm (Buzsaki, 2006; Canolty et al., 2006). Areas and patches of such synchronous activity can be distributed widely across and within the cerebral hemispheres. These and other findings suggest that the physical bases of conscious states consist of spatially dispersed, but reentrantly interconnected, neuronal groups in a widely distributed set of brain areas (Baars, 1988; Edelman, 2003 ) constituting a "Global Workspace.” Evidence also suggests that each separate area functions to process a distinctive feature of the overall conscious scene, and that synchrony serves to "bind" these features together into an apparently seamless whole (Engel and Singer, 2001).

\section{THE ROLE OF BRAIN THEORY}

The biological account presented above raises the problem of describing how the features of consciousness can be related to brain structure and dynamics in a manner that is consistent with evolution. A theory of human consciousness, therefore, must rest on a more global theory of how vertebrate brains are organized to yield function.

To contribute to our understanding of consciousness, such a global brain theory must be in accord with the extraordinary variety of the contents of consciousness. These contents are constructed from both internal memories and external stimuli, and necessarily reflect the complexity, ambiguity, and unpredictability of signals received from encounters with the world. Sampling and integrating that complexity requires brain mechanisms that can deal with endless and unforeseen novelty. Given the implausibility of evolving an instructive mechanism to govern this complex and variable process, it is necessary to invoke a selectional theory. Neural Darwinism, or the theory of neuronal group selection, is just such a theory (Edelman, 1987, 1993). It maintains that the brain gives rise to repertoires of variant neuronal groups of vast complexity and diversity. Selection from these repertoires of neuronal groups occurs to match the novelty and diversity of experience in an integrative and adaptive fashion.

Neural Darwinism has three tenets:

(1) Developmental selection - During the development of the brain, neurons that fire together wire together. While there are a number of genetic constraints on the formation of brain circuits, a number of epigenetic processes leads to extensive individual variance. These circuits constitute a primary repertoire for further selection.

(2) Experiential selection - During development and after the formation of variant neuroanatomy, changes in synaptic strength result in the further selection of variant neuronal groups that is characteristic of individual experience, constituting a secondary repertoire. The distribution and magnitude of these changes are constrained by inborn value systems, a diverse set of neural circuits producing various neuromodulators selected over evolutionary time.

(3) Reentry - Long-range, reciprocal, and massively parallel connections from one brain area to another provide the dynamic spatiotemporal coordination in circuits of groups that is necessary for integrated and adaptive conscious behavior.

\section{EXTENSION OF NEURAL DARWINISM TO CONSCIOUSNESS}

As we have seen, the anatomy of the thalamocortical system provides an essential element in the neural mechanism underlying consciousness. A characteristic feature of the cerebral cortex is the presence of corticocortical connections linking various neurons in spatially dispersed regions of the cortex to one another in a reciprocal fashion (Fuster, 2008). Similarly, the thalamus projects a large number of axons to all areas of the cortex, and the cortex projects an even larger number to the thalamus (Jones, 2007). Together the corticocortical, corticothalamic, and thalamocortical connections provide a necessary structural basis for dynamic reentry, the ongoing reciprocal signaling within the cortex and between the cortex and the thalamus, constituting a Dynamic Core (Edelman and Tononi, 2000). Reentrant coupling can result in the formation of synchronous time-locked patterns of activity essential to connecting and integrating the distinctive functions of different brain areas. Reentrant activity allows a brain area having responses originally evoked by sensory input to give similar responses in the absence of that input. By this means the brain "speaks to itself," a necessary basis for memory and thought.

We have proposed earlier (Edelman, 1989) that reentry between posterior, modality-specific cortical areas and more anterior areas related to memory and executive functions provides a mechanism for conscious processes. The observation that this Dynamic Core (Edelman and Tononi, 2000) necessarily involves integration of activity in widespread distributed cortical areas accords with the concept of a Global Workspace. Experimental evidence for a role for a Dynamic Core of cortical reentrant activity in conscious 
perception has been provided by studies of electromagnetic signals generated in the brains of subjects viewing two different, fluctuating stimuli, one in each eye (Srinivasan et al., 1999). At any time, the subject exhibits binocular rivalry, i.e., is conscious of only one percept; this alternates to the other percept every few seconds. As illustrated in Figure 1, conscious perception of a stimulus is correlated with enhanced coherence among signals from multiple distant cortical areas constituting a Global Workspace. Presumably this synchrony is generated and maintained by the reentrant neural activity that contributes to the Dynamic Core.

Hierarchical recursive mapping by means of reentrant interactions in the Dynamic Core provides a mechanism for the integration of diverse neural signals from widespread cortical areas that give rise to such integrated patterns of neural activity. At any given moment, a process of integration of collective neuronal activity generates an interwoven pattern of responses unique to a particular animal at that particular moment of time (Edelman and Tononi, 2000).

Dynamic changes in the core lead to a linked sequence of discriminations. It has been proposed that the sequences of integrated activity in this discriminatory web give rise to the unitary conscious scenes that constitute phenomenal experience (Edelman, 1992). These temporal properties underlie what William James called the "stream of consciousness" (James, 1890). It has been suggested that a measure of the integration of information accounts for the presence of conscious experience (Tononi, 2005). However, no measure of integration alone can provide an exclusive account of the actual mechanisms that entail phenomenal conscious experience (Seth et al., 2006). Indeed, a number of other properties remain to be accounted for, requiring causal analysis of biological mechanisms. These include not only the temporal properties just mentioned, but also the intentionality or reference of conscious states (Brentano, 1973), as well as their modulation by attention (Knudsen, 2007).

\section{THE GLOBAL WORKSPACE AND CONSCIOUSNESS}

As a result of the growth and integration of cognitive and neuroscientific evidence, it is now evident that Global Workspace theory (Baars, 1988, 2002) is complementary to the Dynamic Core hypothesis. Global Workspace theory reconciles the limited capacity of momentary conscious content with the vast repertoire of long-term memory. A global broadcast of focal conscious contents can be viewed as a momentary "snapshot" of Dynamic Core activity.

The psychological evidence for limited capacity of conscious contents was already very strong in the late 1980s (e.g., Baars, 1988). However, the neurobiological evidence made it equally clear that there are brain-wide phenomena that are also associated with consciousness, such as circadian state changes and event-related potentials. Limitations of the momentary capacity of conscious contents present a puzzle, because intuitively it would seem that multiple conscious contents would allow animals to survive situations in which multiple food sources, or multiple predators, could be tracked simultaneously. Binocular

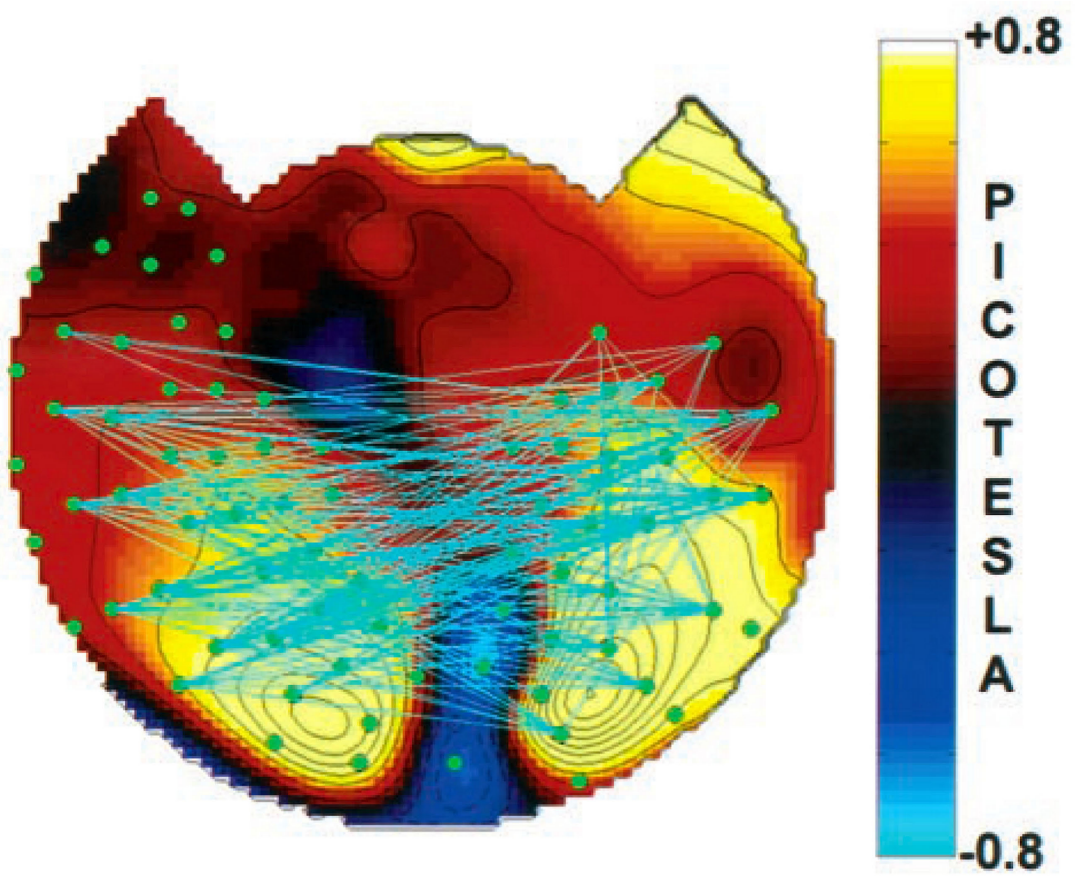

FIGURE 1 | Results of a study of human whole-head magnetoencephalographic (MEG) signals evoked by visual stimuli that were binocularly rivalrous and frequency-tagged (Srinivasan et al., 1999). The topographic map indicates the magnitude of the MEG signal power at the frequency of the perceptually conscious dominant stimulus minus that at the same frequency when the same stimulus was non-dominant and nonconscious. Conscious perception of a stimulus was associated with a significant increase in the calculated coherence between distant MEG channels at the frequency of the perceived stimulus. Pairs of MEG channels whose coherence changed with change in percept are connected in the figure by cyan straight lines. These data point to a role for increased synchrony among distinct and distant neuronal groups in the Global Workspace during conscious perception. Presumably, these widespread groups constituting the Workspace contribute to the Dynamic Core. 
rivalry and binaural selective attention are two famous examples of the inability to integrate incompatible percepts at the same moment. The Global Workspace theory suggests that the limitation on conscious integration has compensatory advantages. For a thirsty giraffe a 300-ms visual percept of a drinking hole may "broadcast" simultaneously to many brain areas, in order to update episodic memory, spatial maps, value systems, prefrontal planning, and motor preparation. In the Dynamic Core at any instant, a single conscious percept may activate numerous brain functions. In a more complex planning task, prefrontal activity involving envisioned goals may similarly prime the Dynamic Core in preparation for a suitable action.

The concept of a Dynamic Core provides a mechanism for Global Workspace events by reentrantly projecting neural signals throughout the cortex. The distributed neural activity that underlies conscious contents simultaneously projects one-way neural signals throughout the cortex. Such directed propagation of activity among widely dispersed large populations of cortical neurons is reflected in event-related potentials. Long-distance propagation of functional brain signals has been known for many years in the context of such potentials (Steriade, 2006). These observations suggest that the concept of a Global Workspace is compatible with the notion that a brief pattern of activity in the Dynamic Core with a specific content, e.g., a particular mental image, momentarily activates numerous cortical areas.

An example is provided by the ventral visual stream, which is widely thought to underlie the content of visual consciousness (Zeki, 1993). The evidence is strong that activity correlated with this stream is widely distributed throughout neocortical and medial temporal lobe pathways. There is direct evidence for such widespread propagation of neural events underlying visual consciousness (Dehaene and Naccache, 2001; Doesburg et al., 2009). Particularly strong support for Baars' proposal comes from the studies of Gaillard et al. (2009). Moreover, some prefrontal regions have been shown to contain visuotopical maps (McGaugh, 2000). These findings are consistent with the involvement of frontoparietal regions in giving rise to the contents of visual consciousness.

There is evidence to suggest the possibility that propagation of signals within the Dynamic Core may occur in integration intervals at a rate of approximately $10 \mathrm{~Hz}$, and that, periodically, the core may switch dynamically from driving one population of neuronal groups in the core to driving another (Canolty et al., 2006). Neural activity required to construct conscious scenes also appears to occur over similar time intervals. Consistent with this time-scale, sensory events in the neocortex underlying conscious percepts are associated with EEG signals in the theta band. Since sensory and motor systems preserve spatiotopical mapping in many brain areas, many oscillatory signals may implicitly encode spatial information as well (Canolty et al., 2006).

\section{THE BEHAVIORAL TRINITY: BRAIN, BODY, AND WORLD}

Two fundamental issues arise in considering the particular brain mechanisms that give rise to consciousness. The first, touched on briefly above, concerns how these biological mechanisms can be related in detail to phenomenal experience (Nagel, 1974; Searle, 1992). The second, and related, issue concerns the basis for the so-called subjective self. To consider these matters from a biological perspective, one must take up the relationships among the brain, the body, and the world (Clark, 1996). For each individual animal, the brain is embodied and the body and the brain are both embedded in the environment. This behavioral trinity cannot be neglected, particularly when considering the evolution and development of the neural structures underlying the conscious process. According to Neural Darwinism (Edelman, 1992, 2003), these forebrain structures were selected for during evolution because they allowed for the planning of adaptive behavior in a complex, changing environment. In responding to any given econiche, the number of possible ways a brain might partition the environment is truly enormous. Furthermore, the complex environment is not a coded piece of tape; it contains dynamic and ambiguous events. As the brain and body respond to diverse sensory inputs and various memory systems, they must remain coordinated with one another.

Dynamic Core activity, with its hierarchical and recursive properties, generates conceptual content by integrating both perceptually driven and motor signals with stored memories. The fundamental tie to world signals in turn provides a basis for intentionality (Brentano, 1973). This proposed mechanism also allows for modulation of core activity by attentional signals arising in subcortical structures projecting to the core, e.g., the cerebellum and the basal ganglia (Hikosaka et al., 1998).

Selection in the core occurs under the further constraint of neuromodulatory value systems located in the basal brain and brain stem. These diffuse ascending systems play a particularly important role in emotional responses, a key aspect of conscious phenomenal experience (Damasio and Dolan, 1999).

\section{QUALIA, SUBJECTIVITY, AND THE SO-CALLED HARD PROBLEM}

How can we account for qualia, subjectivity, and the self? According to the selectional theory based on the behavioral trinity, the experience of qualia occurs in each individual as a set of discriminations: "heat" is not "green," "green" is not "touch," etc. In this view, the complex unified scene at any given moment is a composite of multiple different discriminations integrated within the Dynamic Core.

It has been proposed that no matter how adequately a biological account appears to explain perceptual categorization, memory, and various mechanistic aspects of how the brain works, we remain confounded by the so-called "hard problem": an inability to explain in scientific terms the phenomenal "feel" of conscious experience (Chalmers, 1996). Indeed, many people consider this to be an essential and mysterious problem, one that cannot be solved. Unlike the subjects of other scientific accounts, phenomenal experience entails a first-person point of view, and the suggestion is that it cannot be explained by scientific means. Qualia, the felt contents of consciousness, are therefore concluded to be possibly beyond scientific explanation (Chalmers, 1996). Here, we will attempt to refute this position.

Although the brain and body interact with the external environment, most neural and bodily responses necessarily develop within a single individual's phenotype. Thus, as in the "hard problem" account, qualia are by necessity private. According to Neural Darwinism, qualia reflect higher-order discriminations entailed by the workings of the Dynamic Core (Edelman, 2003). 
For example, to the conscious individual, the experience of blue can be distinguished from the experience of warmth, which can be distinguished from the experience of an odor. No possible description of a phenomenal experience would enable an unequipped individual lacking the proper brain structures, body, or exposure to the appropriate stimuli to have that phenomenal experience. Nonetheless, the correspondence between behavior and report of an individual's qualia as discriminations can, to a large degree, be studied from a third-person point of view. Such a study can be carried out despite the privacy that is an entailed consequence of the properties of the behavioral trinity. It should be added that consciousness itself is not causal (Velmans, 1993; Kim, 2000). It is the neural structures underlying conscious experience that are causal. The conscious individual can therefore be described as responding to a causal illusion, one that is an entailed evolutionary outcome of selection for animals able to make plans involving multiple discriminations.

Once one recognizes that qualia cannot be causal but correspond to internal discriminations that are reliable correlates of underlying causal neural mechanisms, the hard problem as a barrier to scientific explanation disappears. If qualia as discriminations are entailed in behaving agents by the Dynamic Core, in the absence of pathology that entailment is reliable and consistent.

We are left with another set of questions: who are these agents? Exactly "who" experiences qualia in a given body? Or, more succinctly, what is the self? In accordance with the behavioral trinity, the self emerges from brain responses to bodily signals arising in the sensorimotor system of an individual agent. These predominantly motor signals serve to distinguish the body's sense of agency from signals evoked by the movement of another animal's or agent's body. This notion is consistent with the hypothesis that, in sensing agency, motor acts are evaluated internally by comparing signals from a feedforward model of perceptual signals to those arising from the body's motion. In the absence of explicit motion, the same motor components are engaged when evoking an intent to move (Jeannerod, 2001) or where there is a mental "simulation" of an act (Hesslow, 2002). Such a system is constantly active, signaling to the core from the earliest emergence of motor control and throughout the development of proprioceptive "bodily" sense. The combination of such omnipresent sensorimotor signals to the core, and their distinctions that are contemporaneous with perceptual and memorial signals to the core, generates a sense of a self experiencing a surrogate world.

The picture of entailed discriminations proposed here envisions a conscious animal capable of confronting a complex environment full of novelties, and posits that such discriminations give rise to behavior that is adaptive. While certain discriminations may arise unconsciously (Kouider and Dehaene, 2007), they would not, in general, be able to contribute to the planning under novel conditions that incorporates the myriad distinctions made possible by the Dynamic Core and Global Workspace. As long as the illusory sense of conscious causation results in adaptive responses correlated with and informed by appropriate discriminative qualia, there is no issue with describing qualia as causal. Of course, we should recognize that actually they are entailed by neural activity. The foregoing considerations suggest that the hard problem does not require a solution, but rather, a cure.

\section{THE EXPERIMENTAL CHALLENGE AND CONSCIOUS ARTIFACTS}

As we have briefly reviewed, a number of experiments performed in the last decade have shed light on several neural properties underlying conscious experience. The truly hard problem is to provide a biologically based mechanistic account of how, at molecular, cellular, and systemic levels, the brain actually functions to entail consciousness. We propose that core reentry provides the general mechanism for unifying the contents of the Global Workspace, but we are also aware that many important details still remain to be specified.

Given the complexity of the underlying anatomy and physiology, it is not surprising that research on the detailed causal bases of consciousness has trailed far behind evidence for "neural correlates" of consciousness. One approach might mitigate this constraint: namely, building a conscious artifact (Fleischer et al., 2007, in press).

To justify such an enterprise, we must first accept that we are unable in a living animal to trace causal chains at all levels of complexity in the brain circuits that contribute to consciousness. We simply cannot observe, nor causally explore and influence in vivo, the myriad dynamic biological mechanisms underlying conscious processes. Synthetic neural modeling (Reeke and Sporns, 1993) and the construction of brain-based devices provides us, however, with an opportunity to trace causal chains in ways that are not now possible in living animals (Fleischer et al., in press). Given the importance of the behavioral trinity, autonomous exploration of the environment and learning by a brain-based device, driven by a simulated brain incorporating a Global Workspace, a Dynamic Core, and a value system, would be key to success in such an enterprise. A critical benchmark would be the demonstration of imagery, such as mental rotation or "simulation" (Hesslow, 2002), as suggestive of consciousness in the functioning of such an artifact.

A sticking point in such an endeavor relates to the number of simulated neurons and synapses that might be both necessary and sufficient in any synthetic neural model or conscious artifact. If it is necessary to simulate a workspace containing multiple millions of neurons and billions of synapses, modeling may be constrained by limits on processing speed and active memory capacity. But, so far, it appears that a much smaller number of simulated neurons and synapses (Fleischer et al., 2007) might prove sufficient to give rise to a particular mental property, such as imagery.

\section{CONCLUSION AND EXTENSIONS}

Consciousness consists of a stream of unified mental constructs that arise spontaneously from a material structure, the Dynamic Core in the brain. Consciousness is a concomitant of dynamic patterns of reentrant signaling within complex, widely dispersed, interconnected neural networks constituting a Global Workspace.

The contents of consciousness, or qualia, are correlates of discriminations made within this neural system. These discriminations are made possible by perceptions, motor activity, and memories all of which shape, and are shaped by, the activity-dependent modulations of neural connectivity and synaptic efficacies that occur as an animal interacts with its world.

The account given here can serve as a basis for formulating a number of unanswered critical questions concerning the regulation of the conscious process. A key question concerns the autonomous 
regulation of the content of consciousness. How does neural activity in the frontal cortex act to modulate attention (Fuster, 2008)? Are similar mechanisms employed to control motor output to yield behavior? What roles do subcortical neural structures play in these processes? Another set of questions concerns the relationship between consciousness and long-term episodic memories (Tulving, 1987). How can we explain the necessity of explicit prior conscious experience for the existence of such memories?

The mechanisms suggested in the present paper were formulated to account for primary consciousness. With the accession of semantic capability and, in our species, true language, higher-order consciousness emerged. By reference to linguistic tokens, humans can divorce themselves from the "remembered present" of primary

\section{REFERENCES}

Austin, G. M., and Grant, F. C. (1956). Physiologic observations following total hemispherectomy in man. Surgery 38, 239-258.

Baars, B. J. (1988). A Cognitive Theory of Consciousness. Cambridge: Cambridge University Press.

Baars, B. J. (2002). The conscious access hypothesis: origins and recent evidence. Trends Cogn. Sci. 6, 47-52.

Bogen, J. E. (1995). On the neurophysiology of consciousness: I. An overview. Conscious. Cogn. 4, 52-62.

Bogen, J. E. (1997). Some neurophysiologic aspects of consciousness. Semin. Neurol. 17, 95-103.

Brentano, F. (1973). Psychology from an Empirical Standpoint. New York: Routledge.

Buzsaki, G. (2006). Rhythms of the Brain. Oxford: Oxford University Press.

Canolty, R. T., Edwards, E., Dalal, S. S., Soltani, M., Nagarajan, S. S., Kirsch, H. E., Berger, M. S., Barbaro, N. M., and Knight, R. T. (2006). High gamma power is phase-locked to theta oscillations in human neocortex. Science 313, 1626-1628.

Chalmers, D. (1996). The Conscious Mind: In Search of a Fundamental Theory. Oxford: Oxford University Press.

Clark, A. (1996). Being There: Putting Brain, Body, and World Together Again. Cambridge: MIT Press.

Crick, F., and Koch, C. (2003). A framework for consciousness. Nat. Neurosci. 6, 119-126.

Damasio,A. R., and Dolan, R. J. (1999). The Feeling of What Happens. New York: Harcourt Brace.

Dehaene, S., and Naccache, L. (2001). Towards a cognitive neuroscience of consciousness: basic evidence and a workspace framework. Cognition 79, 1-37.

Doesburg, S. M., Green, J. J., McDonald, J. J., and Ward, L. M. (2009). Rhythms of consciousness: binocular rivalry reveals large-scale oscillatory network dynamics mediating visual perception. journal.pone.0006142

Edelman, G. M. (1978). "Group selection and phasic reentrant signaling: a theory of higher brain function," in The Mindful Brain: Cortical Organization and the Group-Selective Theory of Higher Brain Function, eds G. M. Edelman and V. B. Mountcastle (Cambridge: MIT Press), 51-100.

Edelman, G.M.(1987).NeuralDarwinism: The Theory of Neuronal Group Selection. New York: Basic Books.

Edelman, G. M. (1989). The Remembered Present. New York: Basic Books.

Edelman, G. M. (1992). Bright Air, Brilliant Fire: On the Matter of the Mind. New York: Basic Books. Darwinism: selection and reentrant signaling in higher brain function. Neuron 10, 115-125.

Edelman, G. M. (2003). Naturalizing consciousness: a theoretical framework. Proc. Nat. Acad. Sci. U.S.A. 100, 5520-5524.

Edelman, G. M., and Tononi, G. (2000). A Universe of Consciousness. New York: Basic Books.

Engel,A., and Singer,W.(2001). Temporal binding and the neural correlates of sensory awareness. Trends Cogn. Sci. $5,16-25$.

Fleischer, J. G., Gally, J. A., Edelman, G. M., and Krichmar, J. L. (2007). Retrospective and prospective responses arising in a modeled hippocampus during maze navigation by a brain-based device. Proc. Natl. Acad. Sci. U.S.A. 104, 3556-3561.

Fleischer,J.G., McKinstry,J.L., Edelman,D. B., and Edelman, G. M. (in press). “The case for using brain-based devices to study consciousness," in Neuromorphic and Brain-Based Robots: Trends and Perspectives, eds H. Wagatsuma and J. L. Krichmar (Cambridge: Cambridge University Press). PLoS ONE 4, e0006142. doi: 10.1371/

Edelman, G. M. (1993). Neural consciousness (Edelman, 1989) without, of course, displacing that fundamental biological mechanism. What emerges as a result of the combination of primary and higher-order consciousness is a narrative capability encompassing past experience and future plans, as well as the ability to be conscious of being conscious. Inner speech can add its elaborations, contributing to what it is like to be human. Establishment of the neural bases of primary consciousness will surely engender new insights into this most challenging scientific domain.

\section{ACKNOWLEDGMENT}

We are grateful to the Neurosciences Research Foundation for its support.
Fox, M. D., and Raichle, M. E. (2007). Spontaneous fluctuations in brain activity observed with functional magnetic resonance imaging. Nat. Rev. Neurosci. 8, 700-711.

Fuster,J. (2008). The Prefrontal Cortex, 4th Edn. San Diego: Academic Press.

Gaillard, R., Cohen, L., Adam, C. Clemenceau, S., Hasboun, D., Baulac, M., Willer, J. C., Dehaene, S., and Naccache, L. (2009). Converging intracranial markers of conscious access. PLoS Biol. 7, e1000066. doi: 10.1371/journal.pbio.1000061

Hesslow, G. (2002). Conscious thought as simulation of behaviour and perception. Trends Cogn. Sci. 6, 242-247.

Hikosaka, O., Miyashita, K., Miyachi, S., Sakai, K., and Lu, X. (1998). Differential roles of the frontal cortex, basal ganglia, and cerebellum in visuomotor sequence learning. Neurobiol. Learn. Mem. 70, 137-149.

Honey, C. J., Sporns, O., Cammoun, L. Gigandet, X., Thiran, J. P., Meuli, R., and Hagmann, P. (2009). Predicting human resting-state functional connectivity from structural connectivity. Proc. Natl. Acad. Sci. U.S.A. 106 2035-2040.

James, W. (1890). The Principles of Psychology. New York: Henry Holt.

Jeannerod, M. (2001). Neural simulation of action: a unifying mechanism for motor cognition. Neuroimage 14 S103-S109.

Jones, E. G. (2007). The Thalamus, 2nd Edn. Cambridge: Cambridge University Press.

Kanwisher, N. (2010). Functional specificity in the human brain: a window into the functional architecture of the mind. Proc. Natl. Acad. Sci. U.S.A. 107, 11163-11170.

Kim, J. (2000). Mind in a Physical World: An Essay on the Mind-Body Problem and Mental Causation. Cambridge: MIT Press.
Knudsen, E. I. (2007). Fundamental components of attention. Annu. Rev Neurosci. 30, 57-78.

Kouider, S., and Dehaene, S. (2007). Levels of processing during non-conscious perception: a critical review of visual masking. Philos. Trans. R. Soc. Lond., B, Biol. Sci. 362, 857-875.

McGaugh, J. L. (2000). Memory - a century of consolidation. Science 287, 248-251.

Metzinger, T. (2003). Being No One: The Self-Model Theory of Subjectivity. Cambridge: MIT Press.

Milner, B., Corkin, S., and Teuber, H.-L. (1968). Further analysis of the hippocampal amnesic syndrome: 14-year follow-up study of H.M. Neuropsychologia 6, 215-234.

Nagel, T. (1974). What is it like to be a bat? Philos. Rev. 83, 435-450.

Norman, K. A., Polyn, S. M., Detre, G. J., and Haxby, J. V. (2006). Beyond mindreading: multi-voxel pattern analysis of fMRI data. Trends Cogn. Sci. 10, 424-430.

Reeke, G. N. Jr., and Sporns, O. (1993). Behaviorally based modeling and computational approaches to neuroscience. Annu. Rev. Neurosci. 16, 597-623.

Searle, T. (1992). The Rediscovery of the Mind. Cambridge: MIT Press.

Seth, A. K., Izhikevich, E., Reeke, G. N. and Edelman G. M. (2006). Theories and measures of consciousness: an extended framework. Proc. Natl. Acad. Sci. U.S.A. 103, 10799-10804.

Srinivasan, R., Russell, D. P., Edelman, G., and Tononi, G. (1999). Increased synchronization of neuromagnetic responses during conscious perception. J. Neurosci. 19, 5435-5448.

Steriade, M. (2006). Grouping of brain rhythms in corticothalamic systems. Neuroscience 137, 1087-1106.

Stookey, B., Scarff, J., and Teitelbaum, M. (1941). Frontal lobectomy in the treatment of brain tumors. Ann. Surg 113, 161-169. 
Tononi, G. (2005). Consciousness, information integration, and the brain. Prog. Brain Res. 150, 109-126.

Tulving, E. (1987). Multiple memory systems and consciousness. Hum. Neurobiol. 6, 67-80.

Velmans, M. (1993). A reflexive science of consciousness. Ciba Found. Symp. 74, 81-91.

Velmans, M., and Schneider, S. (eds). (2007). The Blackwell Companion to Consciousness. Oxford: Blackwell Publishing.
Verschuuren, J., Chuang, L., Rosenblum, M. K., Lieberman, F., Pryor, A., Posner, J. B., and Dolman, J. (1996). Inflammatory infiltrates and complete absence of Purkinje cells in anti-Yo-associated paraneoplastic cerebellar degeneration. Acta Neuropathol. 1, 519-525.

Zeki, S. (1993). Visions of the Brain. New York: Wiley.

Zelazo, P. D., Moscovitch, M., and Thompson,E.(eds).(2007). Cambridge Handbook of Consciousness. New York: Cambridge University Press.
Conflict of Interest Statement: The authors declare that the research was conducted in the absence of any commercial or financial relationships that could be construed as a potential conflict of interest.

Received: 10 November 2010; paper pending published: 03 December 2010; accepted: 04 January 2011; published online: 25 January 2011.

Citation: Edelman GM, Gally JA and Baars BJ (2011) Biology of conscious- ness. Front. Psychology 2:4. doi: 10.3389/ fpsyg.2011.00004

This article was submitted to Frontiers in Consciousness Research, a specialty of Frontiers in Psychology.

Copyright (c) 2011 Edelman, Gally and Baars. This is an open-access article subject to an exclusive license agreement between the authors and Frontiers Media $S A$, which permits unrestricted use, distribution, and reproduction in any medium, provided the original authors and source are credited. 